\title{
Prevención de la infección por Streptococcus del grupo B en gestantes
}

\section{Prevention of Group B Streptococcal Infection in Pregnant Women \\ Prevenção da infecção pelo Streptococcus do grupo B nas mulheres grávidas: Revisão do tema}

Lina María Martínez-Sánchez, BACTER., Esp., MSc. *

Marcos Restrepo-Arango, Est. **

Emmanuel Sánchez-Díaz, Est. **

\section{Resumen}

Introducción: La infección neonatal por Streptococcus del Grupo B en mujeres gestantes es un problema creciente a nivel mundial y tiene múltiples consecuencias para el recién nacido, su prevención impacta directamente la morbimortalidad neonatal. Objetivo: Brindar al lector información relevante sobre la importancia clínica, nuevos métodos de tamizaje y formas de prevención de la infección por Streptococcus del Grupo B en gestantes, que será de utilidad para evitar complicaciones del binomio materno-fetal. Metodología: En esta revisión de la literatura, se estudiaron 53 artículos, abordando evidencia tanto en el ámbito local e internacional, utilizando las bases de datos PubMed, ScienceDirect y Google Scholar, dentro los criterios de búsqueda se tuvo en cuenta el año de publicación, incluyendo artículos que fueron publicados a partir del año 2011. Resultados: La comunidad internacional ha desarrollado guías y planes de prevención; en la actualidad,

\author{
Juan Sebastián Marín-Cárdenas, Est. ** \\ Daniel Gallego-González, Est. ** \\ María Camila Vélez-Peláez, Est. **
}

\footnotetext{
* Bacterióloga, especialista en Hematología y Manejo del Banco de Sangre, magíster en Educación, docente titular, Escuela de Ciencias de la Salud, Facultad de Medicina, Universidad Pontificia Bolivariana, Medellín, Antioquia, Colombia.

** Estudiantes de la Facultad de Medicina, Universidad Pontificia Bolivariana. Grupo Biología de Sistemas, Escuela de Ciencias de la Salud, Facultad de Medicina, Universidad Pontificia Bolivariana, Medellín, Antioquia, Colombia.
}

Correspondencia: María Camila Vélez Peláez, Calle 78B No.72A-109, Medellín, Antioquia, Colombia. Teléfono:+57(4)4936300. E-mail: mariac.velezpel@upb.edu.co 


\section{Abstract}

Introduction: The neonatal infection by Group B Streptococcus in pregnant women is a growing problem worldwide and has multiple consequences for the newborn, so its prevention directly impacts neonatal morbimortality. Objective: To provide the reader with relevant information about the clinical importance, new screening methods and ways to prevent Group B Streptococcus infection in pregnant women which will be useful to avoid complications in the pairing of maternal-fetal. Methodology: In this review of literature, 53 articles were studied addressing local and international evidence by using PubMed, ScienceDirect and Google Scholar databases; within the search criteria the year of publication was taken into account to do so, thus articles published from 2011 were included. Results: The international community has developed guidelines and prevention plans for this infection. Currently, for an adequate prophylaxis and prevention, various methods are proposed such as the screening for expectant mothers, the use of antibiotics during pregnancy and childbirth, and the development of maternal immunization to prevent infections as well. Conclusions: The colonization by Group B Streptococcus in pregnant women and the risk that this entails to the newborn and his/her mother requires constant updating in techniques for screening, prevention and management of it. Numerous advances in these fields of study have been carried out in recent years, and this strengthening and its development will be the key to impact, in a positive way, the maternal-fetal morbimortality. [MartínezSánchez LM, Restrepo-Arango M, Sánchez-Díaz E, MarínCárdenas JS, Gallego-González D, Vélez-Peláez MC. Prevention of Group B Streptococcal Infection in Pregnant Women. MedUNAB 2017; 20(2): 182-189].

Keywords: Streptococcus Agalactiae; Antibiotic Prophylaxis; Bacterial Infections; Secondary Prevention; Vaccines, Synthetic.

\section{Introducción}

Diversas enfermedades pueden afectar a la mujer durante el periodo de gestación, tales como los trastornos hipertensivos, desórdenes metabólicos e infecciones, entre otras, siendo los procesos infecciosos el grupo de patologías más común. De las mujeres gestantes con algún tipo de infección, solo del 5 al $10 \%$ presenta morbilidad por causa febril y tan solo 0.02 casos por cada 1,000 nacidos vivos en Estados Unidos se asocian a mortalidad neonatal (1). La causa de mortalidad más frecuente entre los recién nacidos es la infección neonatal, la cual se manifiesta como bacteriemia/sepsis, neumonía o meningitis y causa alrededor del $23.4 \%$ de las muertes neonatales a nivel mundial (2), lo que demuestra la importancia de la búsqueda e identificación de factores de riesgo y de agentes etiológicos causantes de infección durante el embarazo, con el fin de desarrollar un diagnóstico precoz y brindar tratamientos oportunos.

Entre los mecanismos de transmisión de este grupo de infecciones, se ha identificado que se dan principalmente al

\section{Resumo}

Introdução: A infecção neonatal pelo Streptococcus do Grupo B em mulheres grávidas é um problema crescente no mundo inteiro e tem múltiplas conseqüências para o recémnascido. Sua prevenção afeta diretamente a morbidade e mortalidade neonatal. Objetivo: Oferecer ao leitor a informação relevante sobre a importância clínica, os novos métodos de seleção e as formas de prevenir a infecção por Streptococcus do Grupo B em mulheres grávidas, o que será muito útil para evitar as complicações tanto para a mãe quanto para o feto. Metodologia: Nesta revisão da literatura, foram estudados 53 artigos, ressaltando as evidências tanto no nível local como internacional y se utilizaram os bancos de dados em PubMed, ScienceDirect e Google Scholar. Um dos critérios da pesquisa foi verificar o ano da publicação e incluir somente os artigos publicados a partir de 2011. Resultados: Atualmente, a comunidade internacional desenvolveu diretrizes e planos de prevenção para a profilaxia e a prevenção adequada, propondo diversos métodos como: A triagem materna, o uso de antibióticos durante a gravidez e o parto, além do desenvolvimento das vacinas maternas para prevenir as infecções. Conclusões: A colonização por Streptococcus do Grupo B em mulheres grávidas e o risco que isso implica para o recém-nascido e sua mãe, requer uma atualização constante nas técnicas da triagem, da precaução e do tratamento. São numerosos os avanços realizados, nesta área, nos últimos anos. O seu fortalecimento e desenvolvimento são fundamentais para interferir positivamente na doença e na mortalidade maternofetal. [Martínez-Sánchez LM, Restrepo-Arango M, SánchezDíaz E, Marín-Cárdenas JS, Gallego-González D, VélezPeláez MC. Prevenção da infecção pelo Streptococcus do grupo $B$ nas mulheres grávidas: Revisão do tema. MedUNAB 2017; 20(2): 182-189].

Palavras chave: Streptococcus agalactiae; Antibioticoprofilaxia; Infecções Bacterianas; Prevenção Secundária; Vacinas Sintéticas.

momento del parto, ya que es aquí donde el feto entra en contacto directo con la flora bacteriana de la vagina, vulva y periné, por tanto, la colonización de estas áreas por diferentes microorganismos representa un factor predisponente para la infección en el neonato (2). El agente más ampliamente conocido y estudiado es el Streptococcus del Grupo B (SGB), que a pesar de hacer parte de la flora vaginal normal, aumenta la probabilidad de infección neonatal por transmisión vertical, y consigo las diferentes complicaciones potencialmente mortales ya mencionadas (3); es por esto que la administración de antibiótico intraparto se ha convertido en una de las herramientas más importantes para prevenir la morbimortalidad materna y fetal (3).

Sin embargo, dar antibiótico intraparto a todas las mujeres embarazadas no resulta ser costo efectivo, por esto, y para prevenir las complicaciones de la infección neonatal por SGB, se han utilizado diferentes métodos de laboratorio para diagnosticarlo oportunamente. Actualmente, se recomienda a nivel mundial la realización de cultivo rectovaginal durante las 35 a 37 semanas de gestación, como 
estrategia de tamizaje y dependiendo del resultado se decide si, se da o no antibiótico intraparto como medida de profilaxis (4). Aunque éste es un método efectivo, el cultivo requiere de 24 a 48 horas para marcar como positivo, tiempo que puede retrasar el inicio del tratamiento en algunos casos, por lo cual se ha discutido la posibilidad de aplicar otras pruebas más rápidas que serán discutidas más adelante (4). El objetivo de esta revisión es brindarle al lector una actualización sobre lo más relevante y nuevo acerca de la importancia de la colonización por SGB en gestantes, las técnicas de tamizaje disponibles y su manejo adecuado, dirigido a evitar la aparición de complicaciones del binomio materno-fetal.

\section{Metodología}

La búsqueda de artículos se llevó a cabo teniendo en cuenta los siguientes descriptores DeCS, creados por BIREME en español e inglés: "Streptococcus agalactiae", "Streptococcus Grupo B", "Bacteremia", "Bacteriemia", "Peripartum Period", "Periodo Periparto", "Pregnancy complications", "Infectious" y "Complicaciones Infecciosas del embarazo". Fueron consultadas las bases de datos PubMed, ScienceDirect y Google Scholar, y de los artículos compatibles con los criterios de búsqueda, fueron seleccionados 43 de la base de datos PubMed, 7 de ScienceDirect y 3 de Google Scholar, para un total de 53 artículos. Se encontraron diferentes modalidades de publicaciones científicas como cartas al editor, metaanálisis, boletines descriptivos, reportes de caso y guías para la práctica clínica, de los cuales se incluyó un artículo de cada uno; adicionalmente, se revisaron 26 artículos originales y 22 revisiones de la literatura. Dentro de los criterios de búsqueda se tuvo en cuenta el año de publicación, incluyendo artículos que fueron publicados a partir del año 2011; se decidió por consenso entre los autores incluir dentro de la revisión una publicación del año 1996 debido a la relevancia de su contenido; adicionalmente, se tuvo en cuenta el idioma, incluyendo únicamente artículos en español e inglés, y que contaran con disponibilidad del texto completo en formato PDF.

Para la ejecución de este proceso se contó con la participación de 5 evaluadores, quienes llevaron a cabo el proceso descrito de forma independiente, para poder de esta forma incluir una cantidad apropiada de artículos.

\section{Infección por Streptococcus del Grupo B (SGB)}

Actualmente, el SGB es el agente más frecuentemente encontrado en menores de 3 meses y en mujeres con infecciones intraparto, siendo una causa infecciosa de inicio temprano en neonatos, con una incidencia en 0.25 casos por cada 1,000 nacidos vivos en Estados Unidos, según lo reportado por los Centros para el Control y la Prevención de
Enfermedades (CDC) en 2010 (5). Estudios previos muestran que en algunas mujeres gestantes existe colonización vaginal por SGB, y que la proporción de mujeres colonizadas varía según la región geográfica y otros factores propios de la población estudiada (3). Por ejemplo, en un estudio realizado con 118 mujeres gestantes de Tubarão, Brasil, se encontró 1 de cada 4 pacientes estaba colonizada por SGB (determinado mediante el cultivo positivo entre las 35 y 37 semanas de gestación) y se obtuvo un asociación estadísticamente significativa entre el cultivo positivo y la raza negra $(p=0.046)$, bajos ingresos $(p=$ $0.003)$, fiebre $(p=0.046)$, leucorrea $(p=0.032)$ y uso de antibióticos durante el embarazo $(p=0.02)(6)$.

La colonización o la presencia de una infección en la madre aumenta el riesgo de colonización por SGB, infección y presencia de sepsis en el neonato (2). Además de esto, se ha demostrado que el antecedente de colonización de la madre con este microorganismo predispone a colonizaciones en los embarazos posteriores ( $\mathrm{RR}=2.2, \mathrm{IC} 95 \%: 1.3-3.8)(7)$. También se ha planteado la posibilidad de transmisión a través de la leche materna, sin embargo, los estudios no presentan suficiente evidencia que permita demostrar esta teoría. Por ejemplo, en una cohorte de 160 mujeres de Italia, con sus respectivos hijos recién nacidos, se encontraron 6 mujeres con cultivos de leche materna positivos para SGB y sus hijos, a su vez, fueron identificados como 'fuertemente' colonizados. Sin embargo, todas estas pacientes también presentaban cultivos recto-vaginales positivos, por lo que no fue posible demostrar la causalidad de la lactancia como mecanismo de transmisión (8).

La infección por SGB puede clasificarse como:

- De inicio temprano (0 a 6 días), la cual lleva a sepsis neonatal grave, alteraciones cardiovasculares, manifestaciones respiratorias, meningitis y alteraciones en el neurodesarrollo. Todo lo anterior conlleva a una elevada morbilidad y mortalidad neonatal (9). Un estudio en Polonia encontró una alta incidencia de infección de inicio temprano por SGB entre 910 neonatos con muy bajo peso al nacer (6.6 por cada 1,000 nacidos vivos), con una tasa de mortalidad del $33 \%$ en este grupo de pacientes (10).

- Infección de inicio tardío (7 a 90 días), ésta puede cursar con sepsis, meningitis e infección focal, además, se asocia con lesiones cerebrales neonatales o incluso la muerte. Un estudio de cohorte demostró que se presentan más casos de infección por SGB de inicio tardío en el grupo de los prematuros (incidencia de 1.4 vs. 0.24 por 1,000 nacidos vivos pretérmino y a término, respectivamente), siendo mayor el riesgo en el subgrupo de pacientes nacidos antes de las 34 semanas (pretérmino temprano, $\mathrm{OR}=16.3, \mathrm{IC} 95 \%$ : 10.2-26.2) y mayor el porcentaje de mortalidad $(20 \%$ de neonatos pretérmino vs. $1.5 \%$ a término) en los pacientes con necesidad de soporte con catecolaminas y ventilación mecánica(11). 


\section{Tamizaje}

El primer paso para evitar la infección por SGB es contar con un sistema de tamizaje que permita identificar la población susceptible, para iniciar de forma oportuna la profilaxis antibiótica, reduciendo de esta forma la incidencia de infección temprana en los neonatos (4). Una revisión sistemática realizada por Ohlsson y colaboradores muestra que tras la administración del antibiótico profiláctico se redujo el riesgo de infección en comparación con la no aplicación de algún tratamiento, con un $\mathrm{RR}=0.17$ (IC95\%: 0.04-0.74) (3). Actualmente la prueba de oro es el cultivo de muestra recto vaginal en un medio selectivo, la cual consiste en realizar un hisopado de la región perineal que incluya tejido rectal y vaginal y trasladarlo a un medio selectivo que permite el crecimiento del SGB en una muestra polimicrobiana. Este medio selectivo disminuye en un 50\% los falsos negativos frente a los cultivos no selectivos, alcanzando a tener una sensibilidad del $87 \%$ y una especificidad del $96 \%(12,13)$.

Otro medio utilizado es el subcultivo en agar sangre y la identificación del SGB por el test de CAMP, el cual consiste en la beta hemólisis que se produce en el agar sangre tras la interacción de las sustancias producidas por el SGB y el Staphylococcus aureus en el medio; lastimosamente posee una sensibilidad entre $54-87 \%$ y los resultados pueden tardarse hasta 72 horas (14).

El tiempo requerido para el cultivo es una de las limitaciones actuales que debe tenerse en cuenta, ya que el uso sugerido de antibióticos por vía intravenosa debe iniciarse 4 horas antes del parto y en algunos casos el trabajo de parto puede cursar solo con 2 horas de duración, es por esto que es necesario desarrollar pruebas que permitan obtener resultados confiables en un corto tiempo; entre las propuestas está el uso de la identificación serológica mediante aglutinación de látex con antisueros para el SGB con una sensibilidad y especificidad del 98 y $99.5 \%$, respectivamente (14); Un ejemplo es el ensayo Xpert ${ }$ SGB, $(15,16,17)$ que usa sondas de DNA mediante la amplificación la cadena de la polimerasa con transcriptasa reversa (PCR-RT) (12), lo que permite identificar partes específicas del ácido desoxirribonucleico del SGB, con una sensibilidad del 87\% y una especificidad del 96\% (17). Se ha diseñado también una prueba rápida basada en inmunoblot, en la cual se utiliza una membrana de nitrocelulosa cubierta con anticuerpos específicos para el SGB, con la que se logra identificar la colonización por este microorganismo en un máximo de 6.5 horas, con una sensibilidad del $97.1 \%$ y una especificidad del $88.4 \%$ (18).

El problema que presentan todas las pruebas moleculares anteriores es su elevado costo y que aún no se llega a un acuerdo sobre la relación costo/beneficio que tienen dentro de la práctica clínica (19). Otros autores afirman que el tamizaje debe enfocarse en las pruebas no moleculares, como subcultivo de caldo de zanahoria mejorado, el cual consiste en un medio rico en nutrientes que permite el crecimiento selectivo del SGB y posterior identificación por la gamma hemólisis que provoca en este medio; cabe resaltar que tiene características de rendimiento aceptables para la detección y aislamiento del SGB con una sensibilidad del $82.5 \%$ y una especificidad hasta del 100\% (20).

Otro de los temas discutidos es el momento en que deben realizarse estas pruebas. Generalmente se sugiere que se lleven a cabo entre las semanas 35 y 37 del embarazo, sin embargo, la presencia de factores de riesgo implica un inicio más temprano del seguimiento de la materna (21). Estudios sugieren que se adicione a los protocolos establecidos la detección entre 30 y 32 semanas para las mujeres con factores de riesgo conocidos para el parto prematuro (22).

En conclusión, a pesar del desarrollo de estas nuevas pruebas, en la actualidad el tamizaje se basa en el cultivo recto vaginal.

\section{Uso de antibióticos}

Existen diferentes indicaciones para el manejo de la profilaxis antibiótica para el SGB $(15,17)$ :

- Neonato previo con infección por SGB.

- Bacteriuria por SGB durante cualquier trimestre del embarazo actual.

- Mujeres con cultivo positivo para SGB.

- Estado desconocido sobre el cultivo de SGB, es decir: no se ha hecho el cultivo, está incompleto, o el resultado es desconocido y presenta alguno de los siguientes:

- Parto $<37$ semanas de gestación.

- Rotura de membranas ovulares $\geq 18$ horas

- La temperatura durante el parto $\geq 38.0^{\circ} \mathrm{C}$

- Otra prueba confirmatoria de SGB positiva, diferente al cultivo.

El uso de antibióticos hace parte de las guías para la prevención y control de la infección por SGB, sin embargo, se deben tener múltiples aspectos en consideración, entre ellas el problema actual de la resistencia bacteriana que implica un cuidado especial a la hora de elegir el mejor tratamiento durante el embarazo y el parto. Actualmente, las tasas de resistencia a eritromicina y clindamicina están aumentando $(23,24)$, en un estudio realizado en 700 maternas de la ciudad de Medellín, Colombia, se determinó una resistencia a clindamicina y eritromicina del $28 \%$ por macrólidos lincosamidas y estreptograminas del tipo B (MLSB) inducible y constitutiva (25); además, un estudio de 17 años de duración llevado a cabo en un hospital de Latinoamérica, demostró un aumento en la resistencia a estos dos antibióticos, encontrando $12.5 \%$ para eritromicina y $9.4 \%$ para clindamicina (26), es por esto que no se recomienda su uso sin un antibiograma previo (24). Esta medida también aplica para el uso de macrólidos y lincosamida, por lo que no pueden usarse como terapia empírica (27). 
Estudios internacionales sugieren el uso de penicilina como profilaxis durante el parto (23), y en caso de alergia a este antibiótico y falta de antibiograma, las guías del CDC (Centers for Disease Control and Prevention) recomiendan el uso de cefalosporinas o vancomicina (27). En los casos de alergia a la penicilina deben realizarse pruebas de sensibilidad, sin embargo, se ha reportado que frecuentemente no se siguen las guías de forma apropiada y suele faltar información sobre esta condición en las historias clínicas (28) lo que puede llevar a casos de reacciones anafilácticas durante el parto $(29,30)$.

Otro de los aspectos importantes a tratar es el tiempo de terapia necesario para prevenir la sepsis; se ha demostrado que el uso de betalactámicos por 4 o más horas antes del parto es altamente eficaz para prevenir la infección temprana, mientras que el uso de profilaxis de duraciones más cortas tiene menos eficacia $(30,31)$, aumentando incluso el riesgo de sepsis neonatal cuando el uso es por menos de dos horas (32). Estos estudios también afirman que el uso de betalactámicos como profilaxis antibiótica antes del parto es más efectivo que el uso de clindamicina (32).

A pesar de que se ha demostrado que entre más horas de profilaxis, mayor es la eficacia, se ha visto que si se administra por más de 24 horas, se aumenta la proporción de organismos Gram negativos con resistencia a la ampicilina (33), por lo que el margen de tiempo en el que se da el tratamiento debe tenerse muy en cuenta para asegurar la eficacia, prevenir la aparición de complicaciones mayores y evitar la resistencia. Cuando las penicilinas no son una opción, debe evaluarse la susceptibilidad a eritromicina y clindamicina, en caso de existir resistencia a éstas, el antibiótico de elección es vancomicina $1 \mathrm{~g}$ por vía intravenosa, cada 12 horas hasta el parto (34). Además, se sugiere la administración de antibióticos no solo durante el parto, sino también antes de éste en pacientes con alto riesgo, como aquellas que presentan insuficiencia cervical o protrusión de membranas $(35,36)$.

\section{Vacunación}

La vacunación de las mujeres gestantes es actualmente una forma de prevención de una amplia variedad de enfermedades como el tétanos, tos ferina, difteria, infecciones por neumococo, meningococo, rotavirus, virus del papiloma humano, Haemophilus influenzae tipo b y el herpes zóster $(37,38)$. Sin embargo, en cuanto al SGB, el beneficio de una vacuna comparado con la profilaxis con antibióticos sería que podría prevenir la infección de inicio tardío y no conlleva el riesgo de aumentar la resistencia bacteriana. Vacunas con varios mecanismos de acción diferentes que aseguren un óptimo resultado aún están en desarrollo, en diferentes etapas de experimentación.

El blanco molecular de gran parte de las vacunas es el Polisacárido capsular (CPS, por sus siglas en inglés) con el que se logran diferenciar hasta 10 serotipos; basado en esto se han diseñado vacunas multivalentes contra los serotipos más comúnmente aislados (Ia, Ib, II, III y V) (39), con lo que se lograría cubrir hasta un $85 \%$ de las infecciones por este patógeno (40).

En 1996 se realizó uno de los primeros ensayos clínicos de vacunas conjugadas contra SGB; para este ensayo clínico se utilizó como proteína transportadora el toxoide tetánico, asociado al CPS III, comparada con una no conjugada. Los resultados obtenidos indicaron que no hubo complicaciones en el perfil de seguridad y se produjeron principalmente anticuerpos tipo IgG. El 90\% de las pacientes con vacuna conjugada tuvieron un aumento de 4 veces el valor inicial de anticuerpos, mientras que solo el $50 \%$ de aquellas con vacuna no conjugada lograron este aumento $(p=0.0015)$ (41).

Actualmente, también hay una vacuna trivalente conjugada polisacárido-proteína de SGB (SGB-CV, por sus siglas en inglés), con epítopes capsulares de los serotipos Ia, Ib y III en un estudio fase II (42). Por otro lado, en fase I-II se ha evaluado una vacuna contra el serotipo III que ha demostrado ser tolerada y presentar buena concentración de anticuerpos en el nacimiento y las primeras semanas de vida, pero que requiere más estudios en cuanto a reducción de morbimortalidad e impacto en las complicaciones perinatales (43).

La relevancia de los estudios previamente mencionados, radica en que se ha demostrado que a mayor concentración de IgG anti CPS hay menos desarrollo de enfermedad tardía y temprana por SGB; sin embargo, existen diferentes distribuciones de los serotipos según el tiempo y ubicación geográfica lo que hace más complejo el cubrimiento adecuado de todos los pacientes (40). Por ejemplo, aunque en la mayoría de los aislamientos se encuentran los serotipos más comunes, algunas veces pueden presentarse grupos clonales únicos, lo que sugieren que existe intercambio capsular $(44,45)$, información que ha impulsado al desarrollo de nuevos blancos en el desarrollo de las vacunas, como la peptidasa C5a, proteína Sip y estructuras similares a pili (40). Éstas últimas fueron descritas en un estudio en el que dentro de 898 aislamientos de SGB, el 70\% compartía al menos una isla de pilis, PI142. También se han estudiado proteínas inmunogénicas, tales como la enolasa, aldehído deshidrogenasa y factor de elongación Tu(46).

En el diseño de estas vacunas se debe mantener presente la meta de desarrollarlas lo más inmunogénicas posible, con el objetivo de cubrir también a la población de prematuros y prevenir adecuadamente la infección neonatal. En este aspecto se está trabajando con vacunología reversa y vacunas con glicoconjugados (47); estas últimas aumentan la concentración de anticuerpos y disminuyen la colonización recto-vaginal por SGB, previniendo así la infección invasiva. Gracias a estos mecanismos se ha demostrado que 
no solo se reduce la infección en un $88 \%$, sino que, adicionalmente se previene el parto prematuro y mortinato relacionado con ésta (48). Además de esto, se ha demostrado que tras la buena respuesta a la vacuna glicoconjugada para CPS Ia, II o V, la respuesta inmunogénica continúa por 18 a 24 meses, lo que podría brindar una protección a largo plazo, por lo que se sugiere la evaluación de parámetros inmunogénicos en futuros estudios de efectividad de nuevas vacunas (49).

\section{Conclusiones}

Tanto el fortalecimiento de los protocolos para la profilaxis con antibióticos, como el desarrollo de una vacuna lo más inmunogénica y segura posible, prometen un mejoramiento en el pronóstico materno-fetal, sobre todo en países que no pueden costear o en los que no se implementan los adecuados métodos de tamizaje (50). A pesar del significativo incremento de países que han acogido los protocolos de tamizaje para la detección temprana de colonización por SGB (51), las infecciones neonatales y complicaciones maternas siguen presentándose a nivel mundial, tanto en los países desarrollados como en aquellos en vía de desarrollo. Es por esto, que además de un buen tamizaje, se requieren métodos profilácticos costo efectivos y seguros para la gestante y su hijo, que garanticen el control de la colonización por SGB y consecuentemente lleven a una disminución de la morbimortalidad por secundaria a infecciones por este microorganismo. $(52,53)$.

\section{Conflicto de intereses}

Los autores declaran no tener conflictos de interés.

\section{Referencias}

1. Cape A, Tuomala RE, Taylor C, Puopolo KM. Peripartum bacteremia in the era of group $B$ streptococcus prophylaxis. Obstet Gynec. 2013; 121(4):812-8. Disponible en: http://goo.gl/ZQ41oy .

2. Chan GJ, Lee AC, Baqui AH, Tan J, Black RE. Risk of early-onset neonatal infection with maternal infection or colonization: a global systematic review and metaanalysis. PLoS Med. 2013; 10(8):e1001502. Disponible en: http://www.ncbi.nlm.nih.gov/pubmed/23976885

3. Ohlsson A, Shah VS. Intrapartum antibiotics for known maternal Group B streptococcal colonization. Cochrane Database Syst Rev. 2013; 3:CD007467. Disponible en: http://www.ncbi.nlm.nih.gov/pubmed/23440815

4. Faro J. More rapid testing group B streptococcus detection. MLO Med Lab Obs. 2013; 45(6):15. Disponible en: http://www.ncbi.nlm.nih.gov/pubmed/ 23875438

5. Baker CJ. The spectrum of perinatal group $B$ streptococcal disease. Vaccine. 2013; 31 Suppl 4:D3-6. Disponible en: http://goo.gl/ZYboLp .

6. Kruk CR, Feuerschuette OH, da Silveira SK, Cordazo
M, Trapani Júnior $A$. Epidemiologic profile of Streptococcus agalactiae colonization in pregnant women attending prenatal care in a city of southern of Brazil. Braz J Infect Dis. 2013; 17(6):722-3. Disponible en: http://goo.gl/zvnqtN .

7. Page-Ramsey SM, Johnstone SK, Kim D, Ramsey PS. Prevalence of group B Streptococcus colonization in subsequent pregnancies of group B Streptococcuscolonized versus noncolonized women. Am J Perinatol. 2012; 30(5):383-8. Disponible en: http://goo.gl/9XEpWm.

8. Berardi A, Rossi C, Creti R, China M, Gherardi G, Venturelli C, et al. Group B streptococcal colonization in 160 mother-baby pairs: a prospective cohort study. J Pediatr. 2013; 163(4):1099-104. Disponible en: http://goo.gl/wJ1hGA.

9. Oh W. Early onset neonatal group B streptococcal sepsis Am J Perinatol. 2013; 30(2):143-7. Disponible en: http://www.ncbi.nlm.nih.gov/pubmed/23322392

10. Brzychczy-Wloch M, Wojkowska-Mach J, Helwich E, Heczko PB. Incidence of maternal SGB colonization and neonatal SGB disease among very low birth weight Polish neonates. Med Sci Monit. 2014; 19:34-9. Disponible en: http://goo.gl/ljA2rb .

11. Berardi A, Rossi C, Lugli L, Creti R, Bacchi ML, Lanari M, et al. SGB Prevention Working Group, Emilia-Romagna. Group B streptococcus late-onset disease: 2003-2010. Pediatrics. 2013; 131(2):e361-8. Disponible en: http://goo.gl/b1o0l6.

12. Park JS, Cho DH, Yang JH, Kim MY, Shin SM, Kim EC, et al. Usefulness of a rapid real-time PCR assay in prenatal screening for group B streptococcus colonization. Ann Lab Med. 2013; 33(1):39-44. Disponible en: http://goo.gl/4CQmKm .

13. Yancey MK, Schuchat A, Brown LK, Ventura VL, Markenson GR. The accuracy of late antenatal screening cultures in predicting genital group B streptococcal colonization at delivery. Obstet Gynecol. 1996; 88:811-5.

14. Verani J, McGee L, Schrag S. Prevention of perinatal group B streptococcal disease, 2010. MMWR Recomm Rep. 2010; 59:1-36.

15. Sheehy A, Davis D, Homer CS. Assisting women to make informed choices about screening for Group B Streptococcus in pregnancy: a critical review of the evidence. Women Birth. 2013; 26(2):152-7. Disponible en: http://goo.gl/DqzOZc

16. Guerrero C, Martinez J, Menasalvas A, Blazquez R, Rodriguez T, Segovia M. Use of direct latex agglutination testing of selective broth in the detection of group B streptococcal carriage in pregnant women. Eur J Clin Microbiol Infect Dis. 2004; 23:61-2.

17. Bourgeois-Nicolaos N, Cordier AG, Guillet-Caruba C, Casanova F, Benachi A, Doucet-Populaire F. Evaluation of the Cepheid Xpert SGB assay for rapid detection of group B Streptococci in amniotic fluids from pregnant women with premature rupture of membranes. J Clin Microbiol. 2013; 51(4):1305-6. Disponible en: http://goo.gl/VAE9Qi

18. Faro JP, Bishop K, Riddle G, Ramirez MM, Katz AR, Turrentine MA, et al. Accuracy of an accelerated, culturebased assay for detection of group B streptococcus. Infect Dis Obstet Gynecol. 2013; 2013:367935. Disponible en : http://goo.gl/G0TPd7

19. Poncelet-Jasserand E, Forges F, Varlet MN, Chauleur C, Seffert P, Siani C, et al. Reduction of the use of antimicrobial drugs following the rapid detection of Streptococcus agalactiae in the vagina at delivery by realtime PCR assay. BJOG. 2013; 120(9):1098-108. 
Disponible en: http://www.ncbi.nlm.nih.gov/pubmed/ 23656626

20. Berg BR, Houseman JL, Garrasi MA, Young CL, Newton DW. Culture-based method with performance comparable to that of PCR-based methods for detection of group B Streptococcus in screening samples from pregnant women. J Clin Microbiol. 2013; 51(4):1253-5. Disponible en: http://goo.gl/NimCEG

21. Burns G, Plumb J. GBS public awareness, advocacy, and prevention--what's working, what's not and why we need a maternal GBS vaccine. Vaccine. 2013; 31, Suppl 4:D58-65. Disponible en: http://goo.gl/YR6MKq

22. Horváth B, Grasselly M, Bödecs T, Boncz I, Bódis J. Screening pregnant women for group B streptococcus infection between 30 and 32weeks of pregnancy in a population at high risk for premature birth. Int J Gynaecol Obstetl. 2013; 122(1):9-12. Disponible en: http://goo.gl/ IZW7I5

23. Kimura K, Matsubara K, Yamamoto G, Shibayama K, Arakawa Y. Active screening of group B streptococci with reduced penicillin susceptibility and altered serotype distribution isolated from pregnant women in Kobe, Japan. Jpn J Infect Dis. 2013; 66(2):158-60. Disponible en: http://goo.gl/OPsblL

24. Capanna F, Emonet SP, Cherkaoui A, Irion O, Schrenzel $\mathrm{J}$, Martinez de Tejada B. Antibiotic resistance patterns among group B Streptococcus isolates: implications for antibiotic prophylaxis for early-onset neonatal sepsis. Swiss Med Wkly. 2013; 25:143. Disponible en: http://goo.gl/QZR8kT.

25. Duque CM, Gómez B, Sánchez DM , Uribe OL. Perfil de sensibilidad de $\mathrm{S}$. agalactiae obtenido a partir de muestras de introito vaginal y región perineal de mujeres gestantes de Medellín (Colombia). NOVA. Publicación Científica EN CIENCIAS BIOMEDICAS. 2011; 9(15): Disponible en: http://www.unicolmayor.edu.co/ invest_nova/NOVA/NOVA15_ARTORIG2_PERFIL.pdf

26. Crespo-Ortiz Mdel P, Castañeda-Ramirez CR, RecaldeBolaños M, Vélez-Londoño JD. Emerging trends in invasive and noninvasive isolates of Streptococcus agalactiae in a Latin American hospital: a 17-year study. BMC Infect Dis. 2014; 14:428. Disponible en: http://www.ncbi.nlm.nih.gov/pubmed/25086463

27. Megged O, Assous M, Weinberg G, Schlesinger Y. Inducible clindamycin resistance in $\beta$-hemolytic streptococci and Streptococcus pneumoniae. Isr Med Assoc J. 2013; 15(1):27-30. Disponible en: http://www.ncbi.nlm.nih.gov/pubmed/23484235

28. Paccione KA, Wiesenfeld HC. Guideline adherence for intrapartum group B streptococci prophylaxis in penicillinallergic patients. Infect Dis Obstet Gynecol. 2013; 2013:917304. Disponible en: http://goo.gl/v575Tv

29. Simons FE, Schatz M. Anaphylaxis during pregnancy. J Allergy Clin Immunol. 2013; 130(3):597-606. Disponible en: http://goo.gl/ppJ1Uc

30. Faro J, Katz A, Ramirez M, Turrentine M, Bishop K, Riddle $G$, et al. Rapid test for growth and determination of antibiotic sensitivity of Group B Streptococcus (SGB) in antepartum women. Am J Obstet Gynecol. 2012; 206(1): S268. Disponible en: http://goo.gl/YjP1qU .

31. Fairlie T, Zell ER, Schrag S. Effectiveness of intrapartum antibiotic prophylaxis for prevention of early-onset group B streptococcal disease. Obstet Gynecol. 2013; 121(3):570-7. Disponible en: http://goo.gl/7BPqcz .

32. Turrentine MA, Greisinger AJ, Brown KS, Wehmanen OA, Mouzoon ME. Duration of intrapartum antibiotics for group B streptococcus on the diagnosis of clinical neonatal sepsis. Infect Dis Obstet Gynecol. 2013; 2013:525878. Disponible en: http://goo.gl/FdD7f8

33. Schrag SJ, Verani JR. Intrapartum antibiotic prophylaxis for the prevention of perinatal group B streptococcal disease: experience in the United States and implications for a potential group B streptococcal vaccine. Vaccine. 2013; 28:31. Disponible en: http://goo.gl/2JyCYi .

34. Centers for Disease Control and Prevention. Prevention of Perinatal Group B Streptococcal Disease Revised Guidelines from CDC, 2010. Disponible en: http://www.cdc.gov/groupbstrep/guidelines

35. Bromiker R, Ernest N, Meir MB, Kaplan M, Hammerman C, Schimmel MS, et al . Correlation of bacterial type and antibiotic sensitivity with maternal antibiotic exposure in early-onset neonatal sepsis. Neonatology. 2013;103(1):48-53. Disponible en : http://goo.gl/Wab4ra .

36. Natale F, Brunelli R, Bizzarri B, Castronovo A, De Curtis $M$. Cervical insufficiency: a new issue for guidelines on prevention of perinatal group B streptococcal disease? Pediatrics. 2013; 131(2):e612-5. Disponible en: http://goo.gl/7NqJ5J .

37. Swamy GK, Garcia-Putnam R. Vaccine-preventable diseases in pregnancy. Am J Perinatol. 2013; 30(2):8997. Disponible en: http://goo.gl/KRG4ed .

38. Esposito S, Bosis S, Morlacchi L, Baggi E, Sabatini C, Principi N. Can infants be protected by means of maternal vaccination? Clin Microbiol Infect. 2012; 18 Suppl 5:8592. Disponible en: http://goo.gl/TSKpRR

39. Lindsey B, Kampmann B, Jones C. Maternal immunization as a strategy to decrease susceptibility to infection in newborn infants. Curr Opin Infect Dis. 2013; 26(3):248-53. Disponible en: http://goo.gl/zQfS32 .

40. Melin P, Efstratiou A. Group B streptococcal epidemiology and vaccine needs in developed countries. Vaccine. 2013; 28;31 Suppl 4:D31-42. Disponible en: http://goo.gl/hGvBTZ

41. Kasper DL, Paoletti LC, Wessels MR, Guttormsen HK, Carey VJ, Jennings HJ, Baker CJ. Immune response to type III group B streptococcal polysaccharide-tetanus toxoid conjugate vaccine. J Clin Invest. 1996; 98(10). Disponible en : http://www.ncbi.nlm.nih.gov/pmc/ articles/PMC507681/

42. Madhi SA, Dangor Z, Heath PT, Schrag S, Izu A, Sobanjo-Ter Meulen A, et al . Considerations for a phaseIII trial to evaluate a group B Streptococcus polysaccharide-protein conjugate vaccine in pregnant women for the prevention of early- and late-onset invasive disease in young-infants. Vaccine. 2013; 28;31 Suppl 4:D52-7. Disponible en: http://goo.gl/UWMwP9 .

43. Munoz FM, Ferrieri P. Group B Streptococcus vaccination in pregnancy: moving toward a global maternal immunization program. Vaccine. 2013; 28;31 Suppl 4:D46-5. Disponible en: http://goo.gl/a9u4sc

44. Souza VC, Kegele FC, Souza SR, Neves FP, de Paula GR, Barros RR. Antimicrobial susceptibility and genetic diversity of Streptococcus agalactiae recovered from newborns and pregnant women in Brazil. Scand J Infect Dis. 2013; 45(10):780-5. Disponible en: http://goo.gl//wMHaR

45. Bellais S, Six A, Fouet A, Longo M, Dmytruk N, Glaser P, Trieu-Cuot P, Poyart C. Capsular switching in group B Streptococcus CC17 hypervirulent clone: a future challenge for polysaccharide vaccine development. J Infect Dis. 2012; 206(11):1745-52. Disponible en: http://goo.gl/YPFksR. 
46. Brzychczy-Wloch M, Gorska S, Brzozowska E, Gamian A, Heczko PB, Bulanda M. Identification of high immunoreactive proteins from Streptococcus agalactiae isolates recognized by human serum antibodies. FEMS Microbiol Lett. 2013; 349(1):61-70. Disponible en: http://goo.gl/E75eda.

47. Chen VL, Avci FY, Kasper DL. A maternal vaccine against group B Streptococcus: past, present, and future. Vaccine. 2013; 31 Suppl 4:D13-9. Disponible en: http://goo.gl/ifcr2B .

48. Edwards MS, Gonik B. Preventing the broad spectrum of perinatal morbidity and mortality through group B streptococcal vaccination. Vaccine. 2013; 31Suppl 4:D66-71. Disponible en: http://goo.gl/CCZm1q

49. Edwards MS, Lane HJ, Hillier SL, Rench MA, Baker CJ. Persistence of functional antibodies to group B streptococcal capsular polysaccharides following immunization with glycoconjugate vaccines. Vaccine. 2013; 30(28):4123-6. Disponible en: http://goo.gl/b5q2ff
50. Le Doare K, Heath PT. An overview of global GBS epidemiology. Vaccine. 2013; 31 Suppl 4:D7-12. Disponible en: http://goo.gl/tnjV00 .

51. Koumans EH, Rosen J, van Dyke MK, Zell E, Phares CR, Taylor A,et al . Prevention of mother-to-child transmission of infections during pregnancy: implementation of recommended interventions, United States, 2003-2004. Am J Obstet Gynecol. 2012; $206(2)$ :158.e1-158.e11. Disponible en: http://goo.gl/rdsolo .

52. Johri AK, Lata H, Yadav P, Dua M, Yang Y, Xu X, et al. Epidemiology of Group B Streptococcus in developing countries. Vaccine. 2013; 31 Suppl 4:D43-5. Disponible en: http://goo.gl/ucTuO5

53. Dagnew AF, Cunnington MC, Dube Q, Edwards MS, French N, Heyderman RS,et al. Variation in reported neonatal group $\mathrm{B}$ streptococcal disease incidence in developing countries. Clin Infect Dis.2012; 55(1):91-102. Disponible en: http://goo.gl/tPJvkl . 